\title{
Draft Genome of Multidrug-Resistant Klebsiella pneumoniae 223/14 Carrying KPC-6, Isolated from a General Hospital in Malaysia
}

\author{
Norazah Ahmad ${ }^{1 \#, ~ T e i k ~ M i n ~ C h o n g 2 \#, ~ R o h a i d a h ~ H a s h i m ㄹ, ~ S u r i a n t i ~ S h u k o r ~}{ }^{1}$, Wai-Fong Yin², Kok-Gan \\ Chan $^{2} \bowtie$ \\ 1. Bacteriology Unit, Institute for Medical Research, Jalan Pahang, 50588 Kuala Lumpur, Malaysia \\ 2. Division of Genetics and Molecular Biology, Institute of Biological Sciences, Faculty of Science, University of Malaya, Kuala Lumpur \\ 50603, Malaysia. \\ \# Both authors contributed equally.
}

$\triangle$ Corresponding author: Division of Genetics and Molecular Biology, Institute of Biological Sciences, Faculty of Science, University of Malaya, Kuala Lumpur 50603, Malaysia; E-mail: kokgan@um.edu.my

(C) 2015 Ivyspring International Publisher. Reproduction is permitted for personal, noncommercial use, provided that the article is in whole, unmodified, and properly cited. See http://ivyspring.com/terms for terms and conditions.

Published: 2015.11.16

\begin{abstract}
We performed whole genome sequencing on a clinical multidrug-resistant Klebsiella pneumoniae strain 223/14. Investigation into its draft genome revealed the presence of KPC-6 variant, suggesting carbapenemase is present in this isolate. We found a plasmid-borne KPC gene (882 bp) inserted between two transposase genes in the genome of $K$. pneumoniae 223/14.
\end{abstract}

Key words: Klebsiella pneumoniae Carbapenemase (KPC), multidrug resistance, whole genome sequencing

The antibiotic Carbapenem is considered a last option to treat nosocomial infections caused by extended spectrum $\beta$-lactamases (ESBL)-producing multidrug-drug-resistant Gram-negative bacteria (1). However, the emergence and widespread of carbapenem resistant pathogens pose a major threat in global healthcare (2). Among the documented resistant mechanisms, increasing worldwide reports of hospital outbreaks caused by Klebsiella pneumoniae Carbapenemase (KPC) producing Enterobacteriaceae are of great concern (3). Known to be the most common carbapenemase, KPC is mostly found in Klebsiella pneumoniae with increasing identification in other Enterobacteriaceae that display carbapenem resistance (4). $\mathrm{KPCs}$ are categorized into eleven known variants (KPC-2 to KPC-12) differing by changes in a few amino acids (5). In this study, the genetic determi- nants for multidrug-resistant of a K. pneumoniae strain $223 / 14$, isolated from infected laparotomy wound of a patient were investigated by whole-genome sequencing.

The genome sequence of $K$. pneumoniae strain 223/14 was generated using Single Molecule Real-Time (SMRT) RSII sequencing platform (Pacific Biosciences, Inc., CA). Prior to sequencing analysis, the genomic DNA was isolated using MasterPure ${ }^{\mathrm{TM}}$ DNA purification kit (Epicentre, USA) followed by shearing of the DNA to fragment size targeted at $10 \mathrm{~kb}$. The SMRTbell library preparation was subsequently performed using Template Preparation Kit (Pacific Biosciences, Inc., CA) and P4/C2 chemistry was employed for the sequencing analysis. A total of 3 SMRT cells were used for sequence collection and the duration was set at 180 minutes per cell with stage 
start option. After acquisition of the sequence data, quality filtering and assembly was subsequently performed using the Hierarchical Genome-Assembly Process (HGAP) module available from the Pacific Biosciences's SMRT portal (6).

A total of 106,678 polymerase reads with average read length of $5,218 \mathrm{bp}$, amounting to $556,747,120 \mathrm{ba}-$ ses were generated after quality filtering process. The polished assembly size of the draft genome was at $6,165,397$ bp with average $G+C$ content of $57 \%$. The assembly has yielded 13 contigs with average sequencing depth of $75.73 \times$ and the largest contig size was at 5,584,553 bp. Using Rapid Annotation Subsystems Technology (RAST) server (7), prediction and annotation of genes in the assembled sequences was performed, identifying 5,973 open reading frames and 112 coding sequences of rRNAs and tRNAs. Validation of ORFs associated with antibiotics resistance was subsequently performed via sequence comparison with NCBI-NR database using BLAST.

A plasmid-borne KPC gene (882 bp) inserted between two transposase genes was identified in the genome of K. pneumoniae 223/14. Sequence alignment using ClustalW (8) with eleven known KPC variants has verified that the gene belongs to KPC- 6 variant based on nucleotide variations at position 147, 308, 502,716 , and 814 of the gene (5). Such finding has represented the emergence of pathogens harboring KPC-6 in Malaysia since the initial detection of this KPC variant from Puerto Rico in 2008 (9).

\section{Nucleotide sequence accession number}

This Whole Genome Shotgun project has been deposited at DDBJ/EMBL/GenBank under the accession JRTV00000000. The version described in this paper is the first version, JRTV01000000.

\section{Acknowledgement}

The authors would like to thank the Director of Health Malaysia for permission to publish this paper. This project was financially supported by University of Malaya-Ministry of Higher Education High Impact Research Grants, (UM-MOHE HIR Grant UM.C/625/ 1/HIR/MOHE/CHAN/14/1, no. H-50001-A000027; UM-MOHE HIR Grant UM.C/625/1/HIR/MOHE/ CHAN/01, no. A000001-50001) which are awarded to KGC.

\section{Competing Interests}

The authors have declared that no competing interest exists.

\section{References}

1. Sacha P, Ostas A, Jaworowska J, Wieczorek P, Ojdana D, Ratajczak J, Tryniszewska E. The KPC type beta-lactamases: new enzymes that confer resistance to carbapenems in Gram-negative bacilli. Folia Histochem Cyto. 2009;47:537-543.

2. Wernli D, Haustein T, Conly J, Carmeli Y, Kickbusch I, Harbarth S. A call for action: the application of the international health regulations to the global threat of antimicrobial resistance. PLoS Medicine. 2011. 8:e1001022.

3. Francis RO, Wu F, Della-Latta P, Shi J, Whittier S. Rapid detection of Klebsiella pneumoniae carbapenemase genes in Enterobacteriaceae directly from blood culture bottles by real-time PCR. Am J Clin Pathol. 2012;137:627-632.

4. Tzouvelekis L, Markogiannakis A, Psichogiou M, Tassios P, Daikos G. Carbapenemases in Klebsiella pneumoniae and other Enterobacteriaceae: an evolving crisis of global dimensions. Clin Microbiol Rev. 2012; 25:682-707.

5. Monteiro J, Widen RH, Pignatari AC, Kubasek C, Silbert S. Differentiation of Klebsiella pneumoniae carbapenemase (KPC) variants by pyrosequencing. J Microbiol Methods. 2014;100:42-45.

6. Chin C-S, Alexander DH, Marks P, Klammer AA, Drake J, Heiner C, Clum A, Copeland A, Huddleston J, Eichler EE. Nonhybrid, finished microbial genome assemblies from long-read SMRT sequencing data. Nature Methods. 2013;10:563-569.

7. Aziz RK, Bartels D, Best AA, DeJongh M, Disz T, Edwards RA, Formsma K, Gerdes S, Glass EM, Kubal M. The RAST Server: rapid annotations using subsystems technology. BMC Genomics. 2008;9:75.

8. Larkin MA, Blackshields G, Brown N, Chenna R, McGettigan PA, McWilliam H, Valentin F, Wallace IM, Wilm A, Lopez R. Clustal W and Clustal X version 2.0. Bioinformatics. 2007; 23:2947-2948.

9. Robledo IE. A novel KPC variant, KPC-6, in a Klebsiella pneumoniae (Kp) isolated in Puerto Rico (PR), C2-3738. 48th Annu. Intersci. Conf. Antimicrob. Agents Chemother. (ICAAC)-Infect. Dis. Soc. Am. (IDSA) 46th Annu. Meet. American Society for Microbiology, Washington, DC. 2008. 